\title{
Surgical approach of primary splenic lymphoma: report of a case and review of the literature
}

\author{
Ioanna Konstantiadou • Aikaterini Mastoraki • Ioannis S. Papanikolaou • George Sakorafas • Michael Safioleas
}

Received: 17 May 2009 / Accepted: 19 August 2009

(C) Indian Society of Hematology and Transfusion Medicine 2009

\begin{abstract}
A 80-year-old woman was hospitalized due to anemia and fever. Computed tomography depicted a solitary concentric, sharply marginated lesion of the spleen. Laparotomy identified a creamy-white soft tumor. Histopathological examination confirmed the diagnosis of non-Hodgkin B-cells anaplastic lymphoma. Extended range of pathological manifestations explains the variety of radiological appearances and difficulty in accurate diagnostic process of primary splenic lymphoma.
\end{abstract}

Keywords Primary splenic lymphoma $\cdot$ Clinical presentation · Diagnostic approach $\cdot$ Surgical management

\footnotetext{
I. Konstantiadou · A. Mastoraki · I. S. Papanikolaou •

G. Sakorafas · M. Safioleas

Department of Surgery, Athens University,

Medical School, Attikon University Hospital,

1 Rimini Street, 12462, Chaidari,

Athens, Greece
}

A. Mastoraki $(\square)$

E-mail: dr_kamast@yahoo.gr

\section{Introduction}

Non-Hodgkin Lymphoma (NHL) consists the most common hematopoietic neoplasm representing approximately $4 \%$ of all cancer diagnoses [1]. Clinical manifestations depend on various parameters such as the location of the lymphomatous process, the rate of tumor growth and the function of the organ being compromised or displaced by the malignant procedure. Although splenic involvement in disseminated disease is frequently encountered, primary splenic lymphoma (PSL) remains an uncommon disorder not strictly defined in the literature [2]. Recent publications elucidate a rare nosologic entity with the tumor burden predominately localized in the spleen. Associated infiltration of splenic hilar and extra-abdominal lymph nodes, bone marrow or liver has also been described reflecting the ambiguity of the accepted term. Clinical features of PSL refer to non-specific symptoms and signs including splenomegaly and abdominal discomfort combined with increased sequestration of abnormal cells. Histological discrimination between the diffuse infiltrative pattern and potential nodular involvement could also be accomplished [3, 4]. Diagnostic approach relies on imaging techniques as PSL detection has been achieved in 10-30\% of patients with an otherwise unremarkable physical examination [5]. Despite previous controversy, splenectomy proved the treatment of choice reassuring permanent relief of symptomatic spleen enlargement or reverse cytopenia. Additional administration of chemotherapeutic regimens has been proposed [6]. The majority of patients present with morphologic features and prognostic factors of low grade NHL. Nevertheless, a few cases of diffuse large cell PSL of unfavorable clinical course have been mentioned [7].

Based on one personal observation we report a case of NHL with primary presentation in spleen diagnosed post-splenectomy. Relevant literature is briefly reviewed. 
Clinical features, outcome, and risk factors for PSL development are discussed, while the role of current specific radiographic findings in the early diagnosis and therapeutic management of this serious and potentially lethal disease is emphasized.

\section{Case report}

A 80-year-old woman was admitted to our hospital due to persisting anemia, anorexia and fever. The patient suffered from general malaise and significant loss of weight (BMI: 20) and she described similar intermittent episodes of hematological abnormalities with associated abdominal discomfort over the preceding 6-months. She also referred a past medical history of hypertension, exophtalmos without thyroid gland adenopathy and polymyalgia rheumatica treated with oral methylprednisolone until the temporal improvement of her condition concerning fever and weakness was confirmed. Meticulous clinical examination revealed a firm, tender mass in the left hypohondrium, a palpable liver edge and subicterus of the sclera combined with a recrudescence of symptoms of anemia. Hematological analyses demonstrated a hemoglobin level of $9.4 \mathrm{~g} / \mathrm{dl}$, erythrocyte sedimentation rate (ESR) $110 \mathrm{~mm} / \mathrm{h}, \mathrm{C}$-reactive protein 140 $\mathrm{mg} / \mathrm{l}$, total bilirubin $3.62 \mathrm{mg} / \mathrm{dl}$, conjugated bilirubin 1.34 $\mathrm{mg} / \mathrm{dl}$, and CA-125 $76 \mathrm{U} / \mathrm{ml}$. All other indices as well as peripheral blood smear, bone marrow aspirate and biopsy and immunological profile series including complement levels, anti-nuclear, anti-DNA, anti-ENA, cardiolipin antibodies, rheumatoid factor, collagen cycle and temporal artery biopsy were within normal limits. Markers for hepatotropic viruses and infectious diseases proved negative. Further pathological findings during gastrointestinal endoscopy were absent.

Multiple small bowel fluid levels, indicating intestinal obstruction, were not apparent in the erect abdominal Xrays. Additional contrast enhanced computed tomography (CT) of the abdomen revealed a solitary concentric, sharply marginated lesion measuring $6 \mathrm{~cm}$ at the upper pole of the spleen. The lymphomatous deposit appeared as a homogeneous hypodense mass without calcification. A slightly enlarged liver and mildly abnormal gallbladder appearance without multiple hyperdense lesions suggestive of the presence of calculi was obvious. Additional parenchymal abnormality of the liver was not identified (Fig. 1). Associated lymphadenopathy extended through the branches of the superior mesenteric artery and aorto-caval or para-aortic regions as well as rare complications such as splenic vein thrombosis or gas formation due to necrosis were excluded. A significant increase in tumor size in comparison with previous CT scan was detected. Moreover, MRI investigation depicted a hypodense neoplasm relative to background spleen measuring $4.5 \mathrm{~cm}$ at the upper posterior part. Imaging findings were considered to represent a neoplastic disorder of splenic origin and the diagnosis of concomitant hemangioma, hamartoma or PSL was considered preoperatively.

Acute exacerbation of the symptoms failed to respond satisfactorily to medical treatment and the patient was referred for surgery as the last resort of diagnostic approach. Subsequent laparotomy revealed a large creamy-white soft lesion which seemed to originate from the spleen and presented the typical macroscopic manifestations of lymphomatous tissue (Fig. 2). The tumor extended up to the medial and lateral capsule of the spleen and was adherent to but not invading the left diaphragm and adjacent abdominal wall. Additional dissection documented the presence of an enlarged splenic hilar lymph node. Liver metastases or extensive malignant deposits throughout the peritoneum and the pelvis, probably representing metastatic perito-

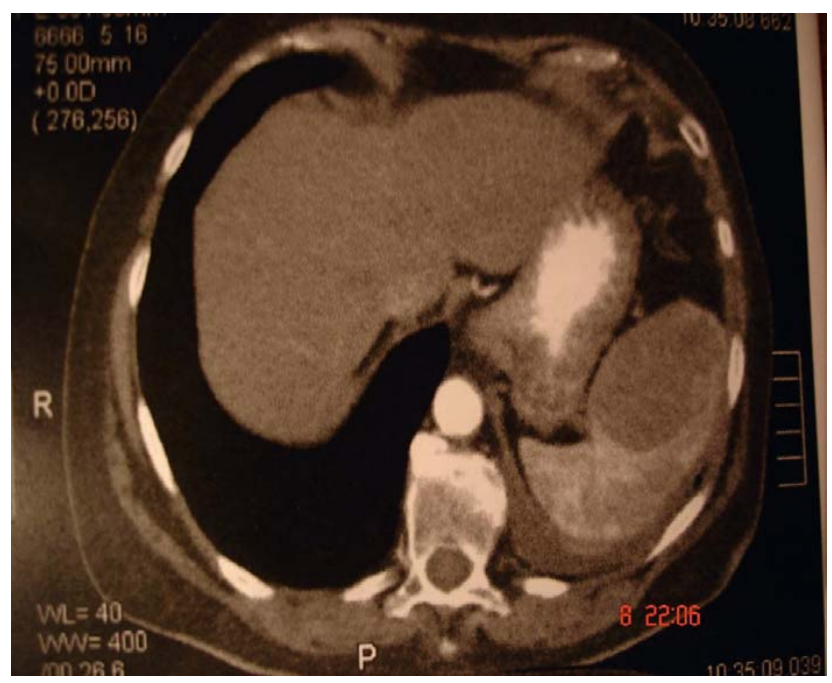

Fig. 1 Depiction of a solitary concentric, sharply marginated lesion measuring approximately $6 \mathrm{~cm}$ at the upper pole of the spleen by contrast enhanced CT of the abdomen

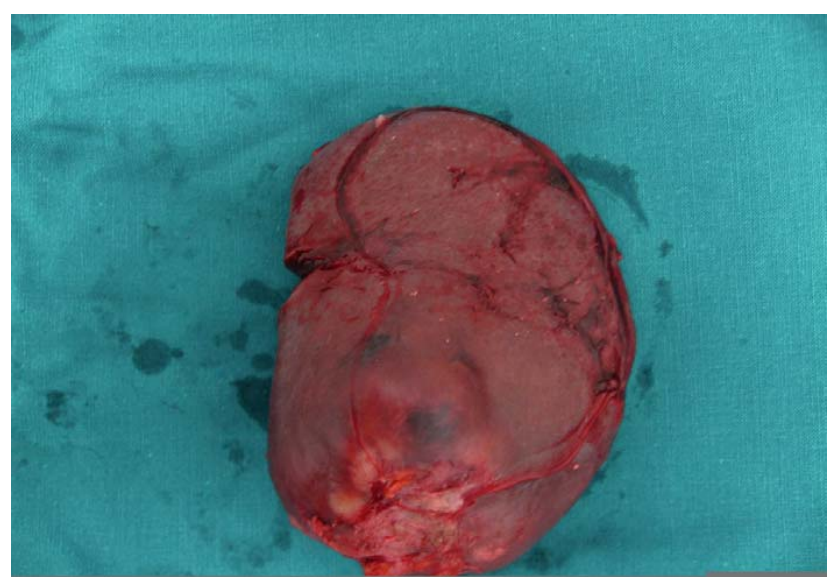

Fig. 2 Intraoperative detection of a large creamy-white soft lesion which seemed to originate from the spleen and presented the typical macroscopic manifestations of lymphomatous tissue 
neal implantations were not elucidated. A liver biopsy was also obtained. Histopathological examination confirmed that the neoplasm, measuring $12 \times 8 \times 5 \mathrm{~cm}$ and weighing $190 \mathrm{~g}$, was diagnosed as a non-Hodgkin B-cells lymphoma of anaplastic type. Additional extensive lymphovascular invasion and metastatic involvement of regional lymph nodes was reported. Synchronous liver biopsy was deprived of diagnostic significance. Immunohistochemistry demonstrated positivity for the epithelial markers CD20 and CD79a, while the neuroendocrine parameters synaptophysin and NSE proved negative. Furthermore, positive staining of neoplastic cells for CD57, desmin and chromogranin was not confirmed. Postoperatively, the patient had an uneventful surgical recovery and was transferred to the oncology department for palliative adjuvant chemotherapy.

\section{Discussion}

NHLs constitute a heterogeneous group of lymphoproliferative malignancies classified seventh in frequency among all human neoplastic disorders. Therapeutic challenge varies greatly depending on tumor stage, phenotype (B-, T- or NK/null-cell), histological picture, clinical behavior, performance status and patients' characteristics along with comorbidities [8]. PSL of non-Hodgkin type consists an extranodal biologically virulent cancer characterized as an aggressive and highly lethal disease. Because of a lack of specific signs or due to its sometime quiet presentation, this condition is frequently diagnosed only at an advanced stage [9]. The subject of PSL has long been controversial issue, since the difficulties in determining whether the malignant lesion develops in the spleen or the organ merely provides a suitable microenvironment for the progression of lymphoid cells have given rise to various diagnostic criteria. In accordance with strict parameters neoplastic invasion is restricted to spleen and associated hilar lymph nodes. Recent additional modification refers to slight potential involvement of liver and/or bone marrow. Enlarged nodes beyond the splenic hilum indicate advanced disease inconsistent with PSL [10]. Extended range of gross pathological manifestations explains the variety of radiological appearances and relative difficulty in accurate radiographic diagnostic process identified in our patient. Meticulous analysis of staging and risk stratification is essential in selecting a treatment modality and predicting the outcome in PSL patients [11]. Finally, according to the original Ahmann classification PSL Group I and II represent localized disease, whereas Group III includes involvement of the liver and nodes beyond the hilum [12].

Because of reported low incidence and its sporadic occurrence it seems difficult to evaluate the clinical features of PSL [13]. Many investigators refer to splenic lymphoma as primary if the predominant feature is splenomegaly or splenic mass with or without associated hypersplenism and/ or B symptoms (temperature $>38^{\circ} \mathrm{C}$, night sweats, weight loss $>10 \%$ from baseline within 6 months). Concomitant painless and slowly progressive peripheral or retroperitoneal adenopathy is often apparent, especially in case of advanced malignant transformation [14]. Direct disease extension beyond the splenic capsule may also occur. Invasion of pancreas, stomach, diaphragm, bowel or greater omentum combined with fistula formation or rapture has also been reported [15]. Nevertheless, in our patient, physical examination revealed neither lympadenopathy nor hepatosplenomegaly. In addition, left upper quandrant pain, which consists the most common presenting symptom, was absent and the patient appeared with an incidental abnormal CT scan combined with persisting anemia, fever, weight loss and fatigue.

Diagnostic approach relies on cross-sectional imaging of the chest, abdomen and pelvis for initial staging, assessing treatment response, and conducting follow-up care. Although the majority of neoplasms are hypoechoic, rare echogenic lesions or bright echoes due to calcifications or gas may be detected. Anechoic areas suggest liquefactive necrosis and in the presence of fever, it may be difficult to distinguish from splenic abscess [16]. Splenomegaly is frequently observed in malignant disorders and can be attributed to potential involvement of the organ, associated hemolytic syndromes, or portal hypertension due to liver dysfunction [17]. In our patient, in spite of accurate determination of splenic size and weight, a firm PSL diagnosis was not promptly implemented by ultrasound. Diffuse infiltration pattern of the spleen was excluded. The sensitivity of the method in the detection of focal parenchymal lesions above $1 \mathrm{~cm}$ is estimated to $54 \%$, while its specificity reaches $100 \%$. Moreover, as the prevalence of local splenic disease is low, hypoechogenic lesions can be interpreted as neoplastic and ultrasound guided fine-needle aspiration (FNA) biopsy is not usually necessary to establish the diagnosis. Our patient had normal splenic size and a $6 \mathrm{~cm}$ hypoechoeic mass in the upper splenic pole combined with no sign of proximal or distal adenopathy was apparent.

It is common knowledge that PSLs are best recognized on contrast enhanced CT scans. Rim enhancement may rarely be encountered indicating pseudocapsule formation or adjacent compressed parenchyma. Profoundly hypodense areas of near water density may represent necrosis or hypovascular solid tumor, possibly due to ischemia. CT scan may also demonstrate spleen size, calcifications and related adenopathy. Infrequent complications such as splenic vein thrombosis, gas formation due to necrosis or superinfection and perisplenic adenopathy combined with gross invasion have rarely been reported. Temporal resolution MRI sequences acquired immediately after intravenous contrast may identify PSL diagnosis. Gallium scan is recommended for detection of initial site of a highly aggressive NHL, evaluation of therapeutic evolution and prediction of 
early lymphoma recurrence [18]. Further post-treatment differentiation of residual disease or relapse from either fibrosis or necrosis can be accomplished using whole body F-deoxyglucose (FDG) positron emission tomography (PET). FNA biopsy of the spleen, although not widely accepted, is gaining renewed interest [19]. Core biopsy, obtained with a 21 or 22 gauge needle or even laparoscopically, has also been used successfully for histological identification of PSL subtype. Nevertheless, histopathological examination of a surgically removed spleen, performed in our case, is essential to confirm the diagnosis.

PSLs are characterized by a distinct immunophenotype which might point to special histogenetic features. Common histological appearance refers to the depiction of multiple nodules occupying the white pulp. When advanced, the entire spleen may be replaced by the tumor, especially of the large cell variety [20]. In some cases, notably in follicular small cleaved lymphoma, the spleen may appear normal with neoplastic cells visible only on high power microscopy. Additional red pulp involvement represents confluence of white pulp disease [21]. Criteria for direct recognition of primary lesions should be morphological including microscopic, micro-nodular constant presence of white and red pulp infiltration and marginal zone pattern with plasmacytic differentiation. Small cleaved, intermediate or large cells with a follicular or diffuse pattern are on occasion present. Histologic subtypes according to the Japanese literature are summarized in the categories of diffuse large cell, lymphoblastic, diffuse small cell and diffuse mixed and lymphoblastic PSL. Concomitant immunohistochemical subdivision of malignant PSL seems to determine associated prognosis and therapeutic outcome [22]. Ahmann classified the gross pathological appearance of primary lymphomatous involvement of the spleen into 4 categories including homogenous enlargement without masses, miliary masses, $2-10 \mathrm{~cm}$ neoplasms and a large solitary tumor. Significant necrosis may occur, particularly in masses increased in size and in histologically defined large cell lymphoma. The irregular, cystic neoplasms may be detected on cross-sectional imaging. Our patient's histopathological sections of spleen revealed NHL of anaplastic large B-cell type with CD20 and CD79a positivity [23].

The differential diagnosis of a solitary splenic mass should include benign entities such as hemangioma, lymphangioma, hamartoma, infarct and abscess as well as metastatic disease. In patients suffering from PSL, lymph node adenopathy is absent and peripheral blood smear is normal, thus the diagnosis may be overlooked. In an asymptomatic patient, the most likely diagnosis of a focal splenic mass is hemangioma with lymphangioma and hamartoma less likely possibilities [24]. Healed infarcts may appear round or irregular shaped findings, whereas acute ischemic lesions are always painful and predisposing cause may be clinically evident. In an afebrile, symptomatic patient, a metastatic disease should be considered and a primary malignancy should be sought [25]. The presence of splenic hilar nodes, a large tumor mass or the unusual complications of the gastrosplenic fistula or splenic vein thrombosis support PSL diagnosis. In the context of an immunocompromised or febrile patient splenic abscess should always be taken into consideration and concomitant lymphoma in case of HIV infection should be excluded.

It has never been clearly stated yet whether splenectomy and/or chemotherapy is effective for the treatment of PSL. Earlier studies concentrate on considerable operative risk during laparotomy in case of advanced disease stage at the time of initial diagnosis. Recently splenectomy has been proposed as a therapeutic intervention, with acceptable morbidity and mortality rates, in each PSL of similar poor prognosis in spite of traditional contraindications [26]. In our patient the operation induced significant reversal of hematological abnormalities. Other investigators suggest that there seemed to be a delay in the correction of anemia in comparison with leucopenia and thrombocytopenia, although a transfusion effect can not be dismissed. On the other hand, once the hematological response is achieved, results appear to be sustained for several months, permitting systemic chemotherapy if necessary. In addition, splenectomy may introduce a prolonged period of disease control and nodal regression, followed by significant favorable survival [27].

Additional chemotherapy represents controversial aspect in the management of PSL, as recent surveys emphasize the role of high-dose treatment in advanced indolent tumors. Early splenectomy eliminates the possibility of local relapse and prevents continuous neoplastic dissemination from the primary site. Thus, the researchers identify that surgical procedures constitute an indispensable point in the treatment of PSL, even though in generalized disease could not ensure significant curative rates and long remission duration [28]. Previous reports on PSL produced an evasive reply regarding the optimal strategy of splenectomy followed by single-agent or combined chemotherapy. Some authors proposed that curability due to splenectomy is irrespective of PSL histologic type. Moreover no significant difference in overall survival between the splenectomized patients treated with adjuvant chemotherapy and those untreated has been mentioned. Based on considerable clinical material and a review of the literature we refer that early splenectomy combined with chemotherapy may serve as the treatment modality of choice for patients presenting with PSL. Splenic irradiation is preferred in case of inoperable neoplasms leading to temporary shrinkage of the spleen and progressive reduction of its size. In our patient splenectomy offered palliation of hypersplenism by removal of tumor burden and correction of hematological disorders at the cost of acceptable surgical risks. Additional chemotherapy combined with the administration of prednisolone reassured patient's recovery. 


\section{References}

1. National Cancer Institute: (1982) The Non-Hodgkin's Lymphoma Pathologic Classification Project. National Cancer Institute sponsored study of classifications of non-Hodgkin's lymphomas. Summary and description of a working formulation for clinical usage. Cancer 49:2121-2135

2. Freeman C, Berg JW, Cutler SJ (1972) Occurrence and prognosis of extranodal lymphomas. Cancer 29:252-260

3. Brox A, Shustik C (1993) Non-Hodgkin's lymphoma of the spleen. Leuk Lymphoma 11:165-171

4. Sumimura J, Miyata M, Nakao K, Kamiike W, Yamaguchi T, Matsuda H (1992) Primary malignant lymphoma of the spleen. Surg Today 22:371-375

5. Singh A, Thapar U, Prabhu R, Naresh K, Joshi A, Supe A (2000) Isolated splenic lymphoma: an elusive preoperative diagnosis. Indian J Gastroenterol 19:184-186

6. Morel P, Dupriez B, Gosselin B, Fenaux P, Estienne MH, Facon T, Jouet JP, Bauters F (1993) Role of early splenectomy in malignant lymphoma with prominent splenic involvement (primary lymphomas of the spleen). A study of 59 cases. Cancer 71:207-215

7. Grosskreutz C, Troy K, Cuttner J (2002) Primary splenic lymphoma: report of 10 cases using REAL classification. Cancer Invest 20:749-753

8. Ambulkar I, Kulkarni B, Borges A, Jagannath P, Advani SH (2006) Primary non-Hodgkin's lymphoma of the spleen presenting as space occupying lesion: a case report and review of literature. Leuk Lymphoma 47:135-139

9. Gobbi P, Grignani G, Pozzetti U, Bertoloni D, Pieresca C, Montagna G, Ascari E (1994) Primary splenic lymphoma: Does it exist? Hematologica 79:286-293

10. Musteata VG, Corcimaru IT, Iacovleva IA, Musteata LZ, Suharschii IS, Antoci LT (2004) Treatment options for primary splenic low-grade non-Hodgkin's lymphomas. Clin Lab Haematol 26:397-401

11. Dachman AH, Buck JL, Krishnan J, Aguilera NS, Buetow PC (1998) Primary non-Hodgkin's splenic lymphoma. Clin Radiol 53:137-142

12. Brox A, Bishinski I, Berry G (1991) Primary non-Hodgkin lymphoma of the spleen. Am J Hematol 38:95-100

13. Kehoe JE, Straus DJ (1988) Primary lymphoma of the spleen: clinical features and outcome after splenectomy. Cancer 62:1433-1438

14. Chacon JL, Mollejo M, Munoz E, Algara P, Mateo M, Lopez L, Andrade J, Carbonero IG, Martinez B, Piris MA, Cruz MA (2002) Splenic marginal zone lymphoma: clinical char- acteristics and prognostic factors in a series of 60 patients. Blood 100:1648-1654

15. Spier C, Kjeldsberg C, Eyre H, Behm FG (1985) Malignant lymhoma with primary presentation in the spleen. Arch Pathol Lab Med 109:1076-1080

16. Troussard X, Valensi F, Duchayne E, Garand R, Felman P, Tulliez M, Henry-Amar M, Bryon PA, Flandrin G (1996) Splenic lymphoma with villous lymphocytes: clinical presentation, biology and prognostic factors in a series of 100 patients. Br J Haematol 93:731-736

17. Gorg C, Weide R, Schwerk WB (1997) Malignant splenic lymphoma: sonographic patterns, diagnosis and follow-up. Clin Radiol 52:535-540

18. Ahmann DL, Kiely JM, Harrison EG, Payne WS (1966) Malignant lymphoma of the spleen. A review of 49 cases in which the diagnosis was made at splenectomy. Cancer 19:461-469

19. Hauke RJ, Armitage JO (2000) Treatment of non-Hodgkin lymphoma. Curr Opin Oncol 12:412-418

20. Falk S, Stutte HJ (1990) Primary malignant lymphomas of the spleen. A morphologic and immunohistochemical analysis of 17 cases. Cancer 66:2612-2619

21. Audouin J, Diebold J, Schvartz H, Le Tourneau A, Bernadou A, Zittou R (1988) Malignant lymphoplasmacytic lymphoma with prominent splenomegaly (primary lymphoma of the spleen). J Pathol 155:17-34

22. Veronessi U, Musumeci R, Pizetti F, Genneri I, Bonadonna G (1974) The value of staging laparotomy in non-Hodgkin lymphomas. Cancer 33:446-452

23. Cavanna L, Artioli F, Vallisa D (1995) Primary lymphoma of the spleen. Report of case with diagnosis by fine needle guided biopsy. Hematologica 80:241-243

24. Kraemer BB, Osborne BM, Butler JJ (1984) Primary splenic presentation of malignant lymphoma and related disorders: a study of 49 cases. Cancer 54:1606-1619

25. Lehne G, Hannisdal E, Langholm R, Nome O (1994) A 10year experience with splenectomy in patients with malignant non-Hodgkin's lymphoma at the norwegian radium hospital. Cancer 74:933-939

26. Hass AC, Brunk SF, Gulesserian HD, Givler I (1971) The value of exploratory laparotomy in malignant lymphoma. Radiol 101:157-165

27. Nakashima A, Nakashima K, Seto H, Kamei T, Kakishita M, Kitagawa M (1994) Primary splenic lymphoma presenting as a large cyst. Radiat Med 12:42-45

28. Mulligan SP, Matutes E, Dearden C, Catovsky D (1991) Splenic lymphoma with villous lymphocytes: natural history and response to therapy in 50 cases. Br J Haematol 78:206-209 